\title{
THE MINIMAL DEGREES OF FAITHFUL REPRESENTATIONS OF THE SPORADIC SIMPLE GROUPS AND THEIR COVERING GROUPS
}

\author{
CHRISTOPH JANSEN
}

\begin{abstract}
The minimal degrees of faithful representations of all sporadic simple groups and their covering groups are determined in this paper.
\end{abstract}

\section{Introduction}

The Brauer character table or p-modular character table of a finite group records, in a concise way, information about the irreducible representations of the group over a field of characteristic $p>0$. For example, this table contains the degrees of all these irreducible representations.

In the last two decades, many of the Brauer character tables for the sporadic simple groups have been calculated. The results for the sporadic groups of orders not exceeding $10^{9}$, the largest of which is the McLaughlin group, have been collected in [20]. Several further results can be found in the literature; a list of references is given at the end of this paper.

When we consider larger groups, the chance of determining the complete table of irreducible Brauer characters decreases. One might be satisfied with partial Brauer character tables, or at least with the degree of the smallest faithful (and therefore irreducible) representation. This will be done here for all sporadic simple groups and their covering groups.

The corresponding problem for ordinary representations (that is, representations over fields of characteristic 0) was settled a long time ago; the results can be found in [2].

\section{The results}

The results are given in Table 1. The first column of this table denotes the name of the group, following the conventions of the ATLAS of finite groups [2]. The second column, with caption 0 , gives the degree of the smallest faithful ordinary representation of the group.

The remaining columns are headed by prime numbers. A column with caption $p$ gives the degree of the smallest faithful representation of the group over a field of characteristic $p$. By general principles, such a representation is necessarily absolutely irreducible (see [24, Chapter 2, Sections 3 and 6]).

Missing entries indicate that $p$ does not divide the order of the group, which implies that the corresponding degree is the same as that over fields of characteristic 0 (see, for example, [24, Chapter 3, Theorem 6.12]). A dash indicates that there is no faithful irreducible representation in this characteristic. Since the Schur multiplier of a sporadic simple group is cyclic, this happens exactly if the group is not simple and $p$ divides the order of its centre. (It is well known that a normal $p$-subgroup of a finite group $G$ is contained in the kernel of every irreducible $p$-modular representation of $G$.) 
Table 1: Minimal degrees of faithful representations for sporadic simple groups and their covering groups.

\begin{tabular}{|c|c|c|c|c|c|c|c|c|c|}
\hline Group & 0 & 2 & 3 & 5 & 7 & 11 & 13 & 17 & 19 \\
\hline $\mathrm{M}_{11}$ & 10 & 10 & 5 & 10 & & 9 & & & \\
\hline $\mathrm{M}_{12}$ & 11 & 10 & 10 & 11 & & 11 & & & \\
\hline $2 \cdot \mathrm{M}_{12}$ & 10 & - & 6 & 10 & & 10 & & & \\
\hline $\mathrm{J}_{1}$ & 56 & 20 & 56 & 56 & 31 & 7 & & & 22 \\
\hline $\mathrm{M}_{22}$ & 21 & 10 & 21 & 21 & 21 & 20 & & & \\
\hline $2 \cdot \mathrm{M}_{22}$ & 10 & - & 10 & 10 & 10 & 10 & & & \\
\hline $3 \cdot \mathrm{M}_{22}$ & 21 & 6 & - & 21 & 21 & 21 & & & \\
\hline $4 \cdot \mathrm{M}_{22}$ & 56 & - & 56 & 56 & 16 & 56 & & & \\
\hline $6 \cdot M_{22}$ & 66 & - & - & 66 & 54 & 36 & & & \\
\hline $12 \cdot \mathrm{M}_{22}$ & 120 & - & - & 48 & 120 & 24 & & & \\
\hline $\mathrm{J}_{2}$ & 14 & 6 & 13 & 14 & 14 & & & & \\
\hline $2 \cdot J_{2}$ & 6 & - & 6 & 6 & 6 & & & & \\
\hline $\mathrm{M}_{23}$ & 22 & 11 & 22 & 22 & 22 & 22 & & & \\
\hline HS & 22 & 20 & 22 & 21 & 22 & 22 & & & \\
\hline $2 \cdot \mathrm{HS}$ & 56 & - & 56 & 28 & 56 & 56 & & & \\
\hline $\mathrm{J}_{3}$ & 85 & 78 & 18 & 85 & & & & 85 & 85 \\
\hline $3 \cdot J_{3}$ & 18 & 9 & - & 18 & & & & 18 & 18 \\
\hline $\mathrm{M}_{24}$ & 23 & 11 & 22 & 23 & 23 & 23 & & & \\
\hline $\mathrm{McL}$ & 22 & 22 & 21 & 21 & 22 & 22 & & & \\
\hline $3 \cdot \mathrm{McL}$ & 126 & 126 & - & 45 & 126 & 126 & & & \\
\hline $\mathrm{He}$ & 51 & 51 & 51 & 51 & 50 & & & 51 & \\
\hline $\mathrm{Ru}$ & 378 & 28 & 378 & 133 & 378 & & 378 & & \\
\hline $2 \cdot \mathrm{Ru}$ & 28 & - & 28 & 28 & 28 & & 28 & & \\
\hline Suz & 143 & 110 & 64 & 143 & 143 & 143 & 143 & & \\
\hline $2 \cdot \mathrm{Suz}$ & 220 & - & 12 & 220 & 220 & 220 & 220 & & \\
\hline $3 \cdot \mathrm{Suz}$ & 66 & 12 & - & 66 & 66 & 66 & 66 & & \\
\hline $6 \cdot \mathrm{Suz}$ & 12 & - & - & 12 & 12 & 12 & 12 & & \\
\hline $\mathrm{O}^{\prime} \mathrm{N}$ & 10944 & 10944 & 154 & 10943 & 406 & 10787 & & & 10944 \\
\hline $3 \cdot \mathrm{O}^{\prime} \mathrm{N}$ & 342 & 153 & - & 342 & 45 & 342 & & & 342 \\
\hline $\mathrm{Co}_{3}$ & 23 & 22 & 22 & 23 & 23 & 23 & & & \\
\hline $\mathrm{Co}_{2}$ & 23 & 22 & 23 & 23 & 23 & 23 & & & \\
\hline $\mathrm{Fi}_{22}$ & 78 & 78 & 77 & 78 & 78 & 78 & 78 & & \\
\hline $2 \cdot \mathrm{Fi}_{22}$ & 352 & - & 176 & 352 & 352 & 352 & 352 & & \\
\hline $3 \cdot \mathrm{Fi}_{22}$ & 351 & 27 & - & 351 & 351 & 351 & 351 & & \\
\hline $6 \cdot \mathrm{Fi}_{22}$ & 1728 & - & - & 1728 & 1728 & 1728 & 1728 & & \\
\hline $\mathrm{HN}$ & 133 & 132 & 133 & 133 & 133 & 133 & & & 133 \\
\hline Ly & 2480 & 2480 & 651 & 111 & 2480 & 2480 & & & \\
\hline Th & 248 & 248 & 248 & 248 & 248 & & 248 & & 248 \\
\hline $\mathrm{Fi}_{23}$ & 782 & 782 & 253 & 782 & 782 & 782 & 782 & 782 & \\
\hline $\mathrm{Co}_{1}$ & 276 & 24 & 276 & 276 & 276 & 276 & 276 & & \\
\hline $2 \cdot \mathrm{Co}_{1}$ & 24 & - & 24 & 24 & 24 & 24 & 24 & & \\
\hline $\mathrm{J}_{4}$ & 1333 & 112 & 1333 & 1333 & 1333 & 1333 & & & \\
\hline $\mathrm{F}_{3+}$ & 8671 & 3774 & 781 & 8671 & 8671 & 8671 & 8671 & 8671 & \\
\hline $3 \cdot F_{3+}$ & 783 & 783 & - & 783 & 783 & 783 & 783 & 783 & \\
\hline $\mathrm{B}$ & 4371 & 4370 & 4371 & 4371 & 4371 & 4371 & 4371 & 4371 & 4371 \\
\hline $2 \cdot \mathrm{B}$ & 96256 & - & 96256 & 96256 & 96256 & 96256 & 96256 & 96256 & 96256 \\
\hline $\mathrm{M}$ & 196883 & 196882 & 196882 & 196883 & 196883 & 196883 & 196883 & 196883 & 196883 \\
\hline
\end{tabular}


Table 1: Minimal degrees of faithful representations for sporadic simple groups and their covering groups (continued).

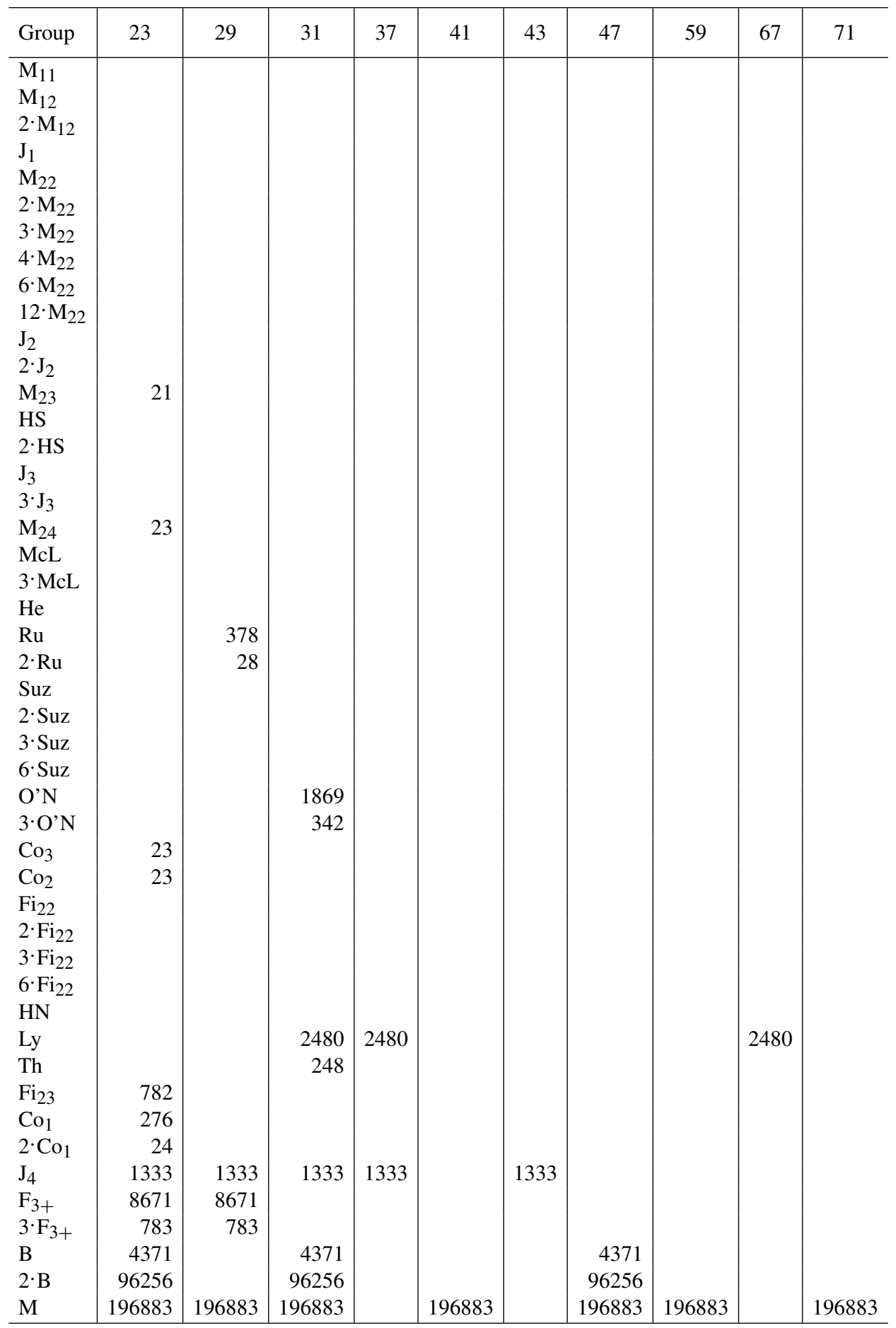




\section{Degrees of minimal faithful Brauer characters}

\section{Cited results}

This section contains references for the cited results that are collected in Table 1.

\subsection{Almost or completely known tables}

The degrees of all irreducible representations for all sporadic groups (and their covering groups) up to Conway's second group $\mathrm{Co}_{2}$ are known, and are contained either in [20] or in GAP; see [3]. Hence we shall start our investigations with the next largest sporadic group, the Fischer group $\mathrm{Fi}_{22}$.

For sporadic groups up to the McLaughlin group, a list of references can be found in [20].

Proofs for the tables for $\mathrm{He}, \mathrm{Co}_{3}$ and $\mathrm{Co}_{2}$ modulo 3 can be found in [16], for He modulo 7 in [26], for Ru modulo 3 in [7] and Ru modulo 5 in [11], for Suz modulo 3 in [17], for O'N modulo 2 and 3 in [19] and O'N modulo 7 in [5], for $\mathrm{Co}_{3}$ modulo 2 in [28], for $\mathrm{Co}_{3}$ modulo 5 in [22], for $\mathrm{Co}_{2}$ modulo 2 in [27, 23], and for $\mathrm{Co}_{2}$ modulo 5 in [13].

The tables for the covering groups of $\mathrm{Fi}_{22}$ and $\mathrm{Fi}_{23}$ modulo 5 can be found in [10] and [12], and the tables for $2 \cdot \mathrm{Co}_{1}$ modulo 7 in [13]. Furthermore, the minimal degrees for $2 \cdot \mathrm{Co}_{1}$ modulo 5 can be determined from the partial decomposition matrices given in [6]. Alternative proofs for the latter two results are given below.

\subsection{The case $p=3$}

The proofs for $\mathrm{Fi}_{22}, 2 \cdot \mathrm{Fi}_{22}, \mathrm{HN}, \mathrm{Ly}, \mathrm{Th}, \mathrm{Fi}_{23}, \mathrm{Co}_{1}, 2 \cdot \mathrm{Co}_{1}, \mathrm{~J}_{4}$ and $\mathrm{F}_{3}+$ modulo 3 have been taken from [16].

\subsection{Brauer trees}

The proofs for the primes dividing the group orders only once can be extracted from [9]. This applies in particular to $\mathrm{Fi}_{22}$ and its coverings modulo 7, 11, 13; to $\mathrm{HN}$ modulo 7, 11, 19; to Ly modulo 7, 11, 31, 37, 67; to Th modulo 13, 19, 31; to $\mathrm{Fi}_{23}$ modulo 7, 11, 13, 17, 23; to $\mathrm{Co}_{1}$ and to $2 \cdot \mathrm{Co}_{1}$ modulo $11,13,23$; to $\mathrm{J}_{4}$ modulo $5,7,23,29,31,37,43$; to $\mathrm{F}_{3+}$ and $3 \cdot \mathrm{F}_{3+}$ modulo 11, 13, 17, 23, 29; to B and to $2 \cdot \mathrm{B}$ modulo 11, 17, 23; and to B modulo 13, 31. There are some cases in $2 \cdot \mathrm{B}$ and $\mathrm{M}$ for which the Brauer trees could not be completely determined in [9]. The proofs for the Monster have been given elsewhere [4], and the proofs for $2 \cdot \mathrm{B}$ are given below.

\subsection{The Monster group}

The result for the Monster group M (and all primes $p$ ) can be found in [4].

\section{The Proofs}

This section contains the proofs for the remaining results.

\subsection{General outline of the proofs}

Most of the following proofs are quite similar in structure. We therefore describe the general idea first. All the computations are performed with (Brauer) characters rather than representations. We use GAP (see [3]) for the calculations.

(i) Upper bounds for the minimal degrees are provided by the degrees of ordinary characters. Every ordinary character of a group $G$ gives rise, through reduction modulo $p$, to 
a $p$-modular character of $G$ of the same degree (see [24, Chapter 3, Subsection 6.2]). Moreover, if $G$ has no nontrivial normal $p$-subgroup, the reduction modulo $p$ of every faithful ordinary character of $G$ is again faithful (by Minkowski's theorem; see [14, Theorem 39.4]). However, the reduction of an ordinary irreducible character is not necessarily irreducible.

For most of the groups in question, faithful representations in most characteristics have been explicitly constructed (see, for example, [2] and [30]). Their degrees give upper bounds for the minimal degrees.

(ii) Lower bounds are provided by restricting a potential character to various suitable subgroups. The restriction to a subgroup $H$ of a faithful character of a group $G$ yields a faithful, but not necessarily irreducible, character of $H$. If the $p$-modular character table of the group $H$ is (partially) known, and if the degree of the restricted character is small, then there are only a few possibilities for the constituents of the restricted character. Looking at several subgroups at the same time, one can drastically reduce the number of possibilities.

The following fusion argument is also commonly used. If two or more conjugacy classes of a subgroup fuse into one conjugacy class of the group, then any restricted character must have the same value on all of these classes. It is here that the library of character tables and the computing facilities of GAP (see [3]) play a significant role.

We shall apply these ideas on a case-by-case basis. All the ordinary and Brauer character tables used in what follows are included in the latest version of GAP. For class names and irrationalities, we follow the notation of [2] and [20].

\subsection{Two sample proofs in detail}

To acquaint the reader with the methods sketched above, we begin with two extended proofs, one for the Harada-Norton group HN in characteristic 3, and one for the Fischer group $\mathrm{F}_{3+}$ in characteristic 2 .

\subsubsection{HN modulo 3}

The ordinary character table of HN and additional information about maximal subgroups and constructions can be found in [2, pp. 164-166]. The character table is available online in GAP, and can be called as follows.

$$
\text { gap> hn := CharacterTable ( "HN" ); }
$$

The list of irreducible characters can be accessed as follows.

gap> irr := Irr ( hn );

The second and third ordinary irreducible characters have degree 133 . We prove below that 133 is the smallest possible degree of a faithful character of $\mathrm{HN}$ in characteristic 3 . This will imply that the ordinary characters of degree 133 remain irreducible modulo 3 .

Let $\varphi$ be a faithful 3-modular character of $\mathrm{HN}$ of degree at most 133 . We restrict $\varphi$ to the maximal subgroup $2 \cdot \mathrm{HS}: 2$ of HN. The GAP-names of the maximal subgroups of $\mathrm{HN}$ can be listed with the following command.

gap> Maxes ( hn );

The ordinary character table of the maximal subgroup $2 \cdot \mathrm{HS}: 2$ is read as follows.

$$
\text { gap> } 2 \text { hs } 2:=\text { CharacterTable ( "2.HS.2" ); }
$$

The fusion map from $2 \cdot \mathrm{HS}: 2$ into $\mathrm{HN}$ is stored on the GAP-character table of $2 \cdot \mathrm{HS}: 2$, and can be accessed by using the following command. 
The fusion map sends the central involution $2 A$ and the class $2 B$ of $2 \cdot \mathrm{HS}: 2$ to $2 A$ of $\mathrm{HN}$. Here, we follow the class notation of [2]; that is, $2 A$ is the first class of elements of order 2 that is the central element in $2 \cdot \mathrm{HS}: 2$, and $2 B$ is the first inverse image of the class $2 A$ of HS:2. The 3-modular character table of $2 \cdot \mathrm{HS}: 2$ can be accessed in GAP as follows.

$$
\text { gap> 2hs2mod3 := CharacterTable( "2.HS.2mod3" ); }
$$

The table can be displayed by the following command.

$$
\text { gap> Display ( 2hs2mod3); }
$$

Since the classes $2 A$ and $2 B$ fuse into one class of $\mathrm{HN}$, the restriction of $\varphi$ to $2 \cdot \mathrm{HS}: 2$ must have equal values on $2 A$ and $2 B$.

The reasoning in the proofs below is supported by a table of the following form. We compare the character values on the classes $2 A$ and $2 B$, and obtain the table below. We include columns that are labelled by the difference between two classes; the values in these columns are obtained by subtracting the corresponding character values on these classes. We use this notation when we want to show that the character values on two particular classes must be equal; that is, the difference must be equal to zero.

\begin{tabular}{rc}
\hline $1 A$ & $2 A-2 B$ \\
\hline $1_{1}$ & 0 \\
$1_{2}$ & 0 \\
$22_{1}$ & 16 \\
$22_{2}$ & 16 \\
98 & 96 \\
$77_{1}$ & 64 \\
$77_{2}$ & 64 \\
$56_{1}$ & -64 \\
$56_{2}$ & -64 \\
\hline
\end{tabular}

The first column contains the degrees, not exceeding 133, of the 3-modular irreducible characters of $2 \cdot \mathrm{HS}: 2$ (characters of equal degrees are distinguished by an index). The next column contains the differences between the character values on the classes as indicated in the heading of the column. The restriction of $\varphi$ to $2 \cdot \mathrm{HS}: 2$ gives rise to a sum of rows of this table with value at most 133 in column one, and zero in the second column.

In GAP we obtain the columns of this table by first transposing the matrix of irreducible Brauer characters, and then subtracting the relevant rows.

$$
\text { gap> modirrtr := TransposedMat ( Irr(2hs2mod3) ); }
$$

As we know the positions of the classes $2 A$ and $2 B$ in the GAP-character table, the column for $2 A-2 B$ can be calculated by the following command.

$$
\text { gap> modirrtr[2] - modirrtr[3]; }
$$

The column for $2 A-2 B$ proves that there must be a $56_{1}$ or $56_{2}$, because these are the only characters with negative value in the column for $2 A-2 B$. Next, either one character of degree 77 , or four characters of degree 22, are contained. The latter case leads to a degree of $56+4 \cdot 22=144>133$. Therefore, the restriction contains $56+77=133$, which proves the result. 


\subsection{2. $\mathrm{F}_{3}+$ modulo 2}

In this proof we obtain the upper bound for the minimal degree from an already-constructed representation. J. Müller has constructed a 3774-dimensional representation for $\mathrm{F}_{3+}$ in characteristic 2 . The existence of this representation was suggested by G. Hiss. We prove that there is no smaller faithful representation.

We consider the maximal subgroup $H:=3^{7} \cdot O_{7}(3)$. Its ordinary and its 2-modular character table can be accessed in [3] by, respectively, the following commands.

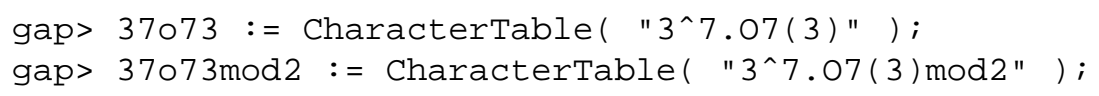

First, we consider the faithful irreducible Brauer characters of $H$. Later on, we shall discuss the characters derived from the factor group $O_{7}(3)$. The only faithful irreducible Brauer characters of $H$ of degrees less than 3774 lead to the following table.

\begin{tabular}{rrrrrrrrr}
\hline $1 A$ & $3 D$ & $3 E$ & $3 D-3 G$ & $3 N$ & $3 N-3 O$ & $3 Q$ & $5 A$ & $21 A$ \\
\hline 728 & -1 & -1 & -54 & 17 & 27 & -1 & 8 & 0 \\
756 & 0 & -27 & -27 & -9 & -27 & 0 & 6 & 0 \\
$2912_{1}$ & -4 & 77 & -54 & 23 & 27 & 5 & -8 & 0 \\
29122 & -4 & -4 & 27 & 23 & 27 & -4 & -8 & 0 \\
\hline
\end{tabular}

The classes $3 D, 3 G, 3 N$ and $3 O$ of $H$ all fuse into $3 D$ of $\mathrm{F}_{3+}$. The classes $3 E, 3 Q, 5 A$ and $21 A$ of $H$ fuse into $3 C, 3 E, 5 A$ and $21 C$ of $\mathrm{F}_{3+}$, respectively. We shall use this information later on, when we rule out the remaining cases by looking at a second subgroup. All the fusion maps mentioned above are stored in the GAP tables.

In order to arrive at a contradiction, we suppose that $\varphi$ is a faithful Brauer character of degree less than 3774 . The column for $3 D-3 G$ proves that the restriction of $\varphi$ to $H$ must contain $2912_{2}$, because this is the only character with positive value in this column. Now, $3 N-3 O$ shows that we have $3668:=2912_{2}+756$ as constituents of $\varphi$, and that no other faithful characters of $H$ occur. Furthermore, $3668(3 D)=-4$ and $3668(3 N)=14$.

We now consider the non-faithful characters of degree less than 106 corresponding to the factor group $\mathrm{O}_{7}(3)$ of $\mathrm{H}$, and we obtain the following values.

\begin{tabular}{rcrrrr}
\hline $1 A$ & $3 D-3 N$ & $3 E$ & $3 Q$ & $5 A$ & $21 A$ \\
\hline 1 & 0 & 1 & 1 & 1 & 1 \\
78 & -9 & -3 & -3 & 3 & 1 \\
90 & -9 & 9 & 9 & 0 & -1 \\
104 & 18 & 23 & -4 & -1 & -1 \\
3668 & -18 & -31 & -4 & -2 & 0 \\
\hline
\end{tabular}

This table implies that the restriction of $\varphi$ must contain a copy of 104 . Hence, we must have $3772:=3668+104$; that is, $\varphi$ has degree 3772 or 3773 , and values $-8+a$ on $3 C$ and $3 E$, $-3+a$ on $5 A$, and $-1+a$ on $21 C$, where $a=0$ in the first case, and $a=1$ in the second case.

To complete our proof, we consider the maximal subgroup He: 2 of $\mathrm{F}_{3+}$. This proof is taken from unpublished work of G. Hiss. 
The fusion of those conjugacy classes that are relevant to the arguments to follow is given in the table below.

\begin{tabular}{c|ccccccc}
\hline He:2 & $1 A$ & $3 A$ & $3 B$ & $5 A$ & $7 B$ & $7 C$ & $21 A$ \\
\hline$G$ & $1 A$ & $3 C$ & $3 E$ & $5 A$ & $7 B$ & $7 B$ & $21 C$ \\
\hline
\end{tabular}

We also list some linear combinations of columns. (Although $3 A$ and $3 B$ fuse into different classes, the character values must be equal, as was proved earlier.)

\begin{tabular}{rrrrrr}
\hline $1 A$ & $3 A-3 B$ & $3 B$ & $7 B-7 C$ & $5 A$ & $21 A$ \\
\hline 1 & 0 & 1 & 0 & 1 & 1 \\
102 & 12 & 0 & 7 & 2 & -2 \\
202 & -18 & 4 & 7 & 2 & 0 \\
492 & -12 & 0 & 0 & -8 & 2 \\
680 & 15 & -1 & -7 & 5 & 0 \\
1920 & 21 & 0 & 7 & -5 & 0 \\
2008 & -3 & -5 & 0 & 8 & -1 \\
\hline
\end{tabular}

A glance at the columns of the above table shows that 1920 is not contained in the restriction of $\varphi$ to He:2, because this would also imply the existence of a copy of 680 (by column $7 B-7 C$ ) and three copies of 492 (by columns $3 A-3 B$ and $7 B-7 C$ ).

The value of $\varphi$ on $5 A$ is negative, and hence 492 occurs. Since 2008 can occur at most once, and since the value of $\varphi$ on $21 A$ is non-positive, it follows that 102 occurs as well. The column $7 B-7 C$ now implies that 680 occurs. Since the value of $\varphi$ on $3 B$ is at most -7 from the above, column $3 B$ proves that 2008 occurs as well. The value on column $3 A-3 B$ is now +12 , and the value on column $7 B-7 C$ is zero. It follows that another 492 must occur. This gives a degree of 3774 , a contradiction.

\subsection{The remaining cases}

\subsection{1. $\mathrm{Fi}_{22}$ modulo 2}

There is a restricted ordinary character of degree 78 that remains irreducible when restricted to $\mathrm{O}_{7}(3)$. This is a minimal faithful character because it is minimal in $\mathrm{O}_{7}$ (3).

\subsection{2. $3 \cdot \mathrm{Fi}_{22}$ modulo 2}

From [30] we know of the existence of an irreducible representation of degree 27. Restriction to $3 \cdot \mathrm{O}_{7}(3)$ shows that it is irreducible as a representation for $3 \cdot \mathrm{O}_{7}(3)$. It is minimal because 27 is the degree of the minimal faithful character for $3 \cdot O_{7}(3)$.

\subsection{3. $\mathrm{Fi}_{22}$ modulo 3}

From [30] we obtain a 77-dimensional representation. Restriction $2 \cdot U_{6}(2)$ shows that 56 is contained, because 56 is the only faithful irreducible 3 -modular representation for $2 \cdot U_{6}(2)$ with degree not exceeding 77 . In turn, it follows that 21 is contained, too. Therefore, 77 is minimal.

\subsection{4. $2 \cdot \mathrm{Fi}_{22}$ modulo 3}

Again, from [30] we obtain a 176 -dimensional representation for $2 \cdot \mathrm{Fi}_{22}$. Any faithful 3modular irreducible representation of $2 \cdot \mathrm{Fi}_{22}$ of degree less than 176 restricts to $2^{2} \cdot U_{6}(2)$, 
as sums of 56 and 120 . The classes $2 C$ and $2 G$ of $2^{2} \cdot U_{6}(2)$ fuse into $2 B$, whereas $2 E$ and $2 \mathrm{H}$ fuse into $2 \mathrm{D}$ of $2 \cdot \mathrm{Fi}_{22}$. We obtain the following results.

\begin{tabular}{rrr}
\hline $1 A$ & $2 C-2 G$ & $2 E-2 H$ \\
\hline 1 & 0 & 0 \\
$56_{1}$ & -32 & 16 \\
$56_{2}$ & 80 & -32 \\
$56_{3}$ & -80 & -32 \\
$120_{1}$ & -160 & -48 \\
$120_{2}$ & 80 & 32 \\
$120_{3}$ & -80 & 32 \\
\hline
\end{tabular}

The column for $2 C-2 G$ proves that a faithful character of $2 \cdot \mathrm{Fi}_{22}$ of degree less than 176 restricts as $56_{2}+56_{3}$. But this contradicts the column for $2 E-2 H$. Hence, 176 is minimal.

\subsubsection{HN modulo 2}

As there is an irreducible ordinary self-dual representation of degree 133, the minimal degree in characteristic 2 is smaller than 133, by Fong's lemma (see [15, Theorem VII.8.13]). The classes $3 A B C$ of $A_{12}$ fuse into $3 A$ of $\mathrm{HN}$, and hence a restriction of a non-trivial character contains $100+16_{1}+16_{2}$.

\subsubsection{HN modulo 3}

See above.

\subsubsection{HN modulo 5}

There is a restricted ordinary character of degree 133 . Restriction to $A_{12}$ (using the classes $3 A B C$, which all fuse into class $3 A$ of $\mathrm{HN}$ ) implies that any character of smaller degree contains 89 and 43 with value -3 on class $3 D$ in $A_{12}$, which fuses into $3 B$ of $H N$. The class $3 C$ of the maximal subgroup $U_{3}(8): 3_{1}$ fuses into $3 B$. But a potential faithful character of degree 132 has a value of least 24 on $3 C$ in $U_{3}(8): 3_{1}$. Therefore, 133 is minimal.

\subsubsection{Ly modulo 2}

We know of the existence of a restricted ordinary character of degree 2480 . We consider the maximal subgroup $G_{2}(5)$. The classes $3 A$ and $3 B$ are mapped to $3 A$ and $3 B$, respectively. The class $5 A$ fuses into $5 A$, and the classes $5 C D E$ fuse into $5 B$. We find the following results.

\begin{tabular}{rrrrr}
\hline $1 A$ & $3 A-3 B$ & $5 A-5 C$ & $5 C-5 D$ & $5 D-5 E$ \\
124 & 0 & 0 & 0 & 0 \\
280 & 24 & -10 & 15 & -5 \\
650 & 24 & 25 & 5 & -10 \\
960 & -48 & -30 & 0 & 0 \\
$1084_{1}$ & 78 & -40 & -5 & -15 \\
$1084_{2}$ & -54 & 95 & -5 & -20 \\
1240 & -24 & -25 & 0 & -15 \\
\hline
\end{tabular}


Assume that $\varphi$ is a Brauer character for Ly, of degree less than 2480. The column $5 D-5 E$ proves that the restriction of $\varphi$ to $G_{2}(5)$ has either 1240 or 650 as a constituent. We consider the following two alternatives.

(i) Assume that the restriction contains exactly one copy of 1240 . Using $5 D-5 E$, we then have 124 or 280 as a constituent of the restriction. If 124 occurs, we prove with $5 C-5 D$ that the degree is at least $1240+124+3 \cdot 960>2480$. On the other hand, if there is a 280 then we have at least $1240+280+960=2480$ (again using $5 C-5 D$ ).

(ii) Assume that we have no 1240 in the restriction. The column $5 D-5 E$ shows that the restriction leads to $m \cdot 650+n \cdot 1$, where $m$ and $n$ are non-negative integers. Restriction to $3 \cdot \mathrm{McL}: 2$ proves that $\varphi(3 A)-\varphi(3 B)$ is divisible by 9 (the fusion map sends $3 B$ to $3 A$ and $3 D$ to $3 B$ ). Therefore, 3 divides $m$. Because $\varphi(1)<2480$, we get $m=3$. But now we have $\varphi(5 A)-\varphi(5 B)=75$ in Ly. Restriction to $3 \cdot \mathrm{McL}: 2$ shows (using the fusions $5 A \mapsto 5 A$ and $5 B \mapsto 5 B$ ) that the degree of $\varphi$ must be at least $15 \cdot 230>2480$.

\subsubsection{Ly modulo 3}

From [18] we obtain a 651-dimensional representation for Ly. Assume that there is a faithful representation with smaller degree. Restriction to $G_{2}(5)$ proves that for each copy of 280 there must be eight copies of 124 (because of $5 A B$ ), which implies that there is no faithful representation of degree 650 or less.

\subsubsection{Ly modulo 5}

This is proved in [21].

\subsubsection{Th modulo 2}

There is an ordinary character of degree 248 . We consider the restriction of a potential Brauer character of degree less than 248 to $H:=\left(3 \times G_{2}(3)\right) .2$. The classes $3 B$ and $3 E$ of $H$ fuse into $3 B$, whereas $3 A, 3 C$ and $3 I$ all go to $3 A$, and $9 A$ and $9 C$ fuse into $9 C$ of Th. We obtain the following values.

\begin{tabular}{rrrrrr}
\hline $1 A$ & $3 A$ & $3 A-3 I$ & $3 A-3 C$ & $3 B-3 E$ & $9 A-9 C$ \\
\hline 1 & 1 & 0 & 0 & 0 & 0 \\
2 & -1 & -3 & 0 & 0 & 0 \\
14 & 14 & 15 & 9 & 9 & 3 \\
28 & -14 & -12 & -9 & 18 & 6 \\
$64_{1}$ & 64 & 66 & 72 & -9 & $-3 z 3$ \\
$64_{2}$ & 64 & 66 & 81 & 0 & $-3 z 3 * *$ \\
78 & 78 & 72 & -91 & 0 & 0 \\
90 & 90 & 90 & -72 & -18 & 0 \\
$128_{1}$ & -64 & -60 & -81 & 0 & $-6 z 3 * *$ \\
$128_{2}$ & -64 & -60 & 171 & 9 & 0 \\
156 & -78 & -90 & -81 & 0 & -6 \\
$180_{1}$ & 180 & 186 & -90 & 9 & 0 \\
$180_{2}$ & -90 & -90 & $-82+27 z 3$ & 9 & -6 \\
$180_{3}$ & -90 & -84 & $-99-27 z 3$ & -6 \\
$180_{4}$ & -90 & -84 & -62 & \\
\hline
\end{tabular}


First, the column $3 A-3 C$ proves that neither $180_{3}$ nor $180_{4}$ can be contained in the restriction of a potential character of degree less than 248 . Next, if $180_{2}$ is a constituent of the restriction, $3 A-3 C$ proves that one copy out of 78,90 or $180_{1}$, or at least nine copies of 14 , or both of $64_{1}$ and $64_{2}$ (look at $9 A-9 C$ ), are part of the restriction. All these possibilities lead to a character whose degree exceeds 247 . Suppose, now, that the restriction contains $180_{1}$. Then none of $128_{1}, 128_{2}$ or 156 can be contained, because of the degree. Hence a non-negative linear combination of 28 and 2 must be contained (look at $3 A-3 I)$. But then we are stuck positive on $3 B-3 E$. Therefore, no 180 is contained in the restriction of a potential character. Assume next that $128_{1}$ is contained in the restriction. But then $9 A-9 C$ implies that we have either $128_{2}$ or $2 \cdot 64_{1}$, a contradiction. We assume now that the restriction contains a 64 . Then the columns for $3 B-3 E$ and $9 A-9 C$ prove that we have $64_{1}+64_{2}+2 \cdot 14$ or $64_{1}+64_{2}+28$. But now we are stuck positive on $9 A-9 C$. The same argument applies when assuming that the restriction contains a 14 or a 28. Therefore, the restriction to $H$ contains only copies of $1,2,78,90$ and 156 . Next, $3 A-3 I$ and $3 A-3 C$ prove that there is $156+90$ in the restriction. Clearly, a potential 247 would restrict as $156+90+1$, with value 13 on $3 A$ of Th.

Let $K:={ }^{3} D_{4}(2): 3$. The classes $7 A$ and $7 B$ both fuse into $7 A$ of $\mathrm{Th}$, and $3 A$ and $3 C$ fuse into $3 A$. We obtain the following table.

\begin{tabular}{rrrc}
\hline $1 A$ & $3 A$ & $3 C$ & $7 A-7 B$ \\
\hline $1_{1}$ & 1 & 1 & 0 \\
$1_{2}$ & 1 & $z 3$ & 0 \\
$11_{3}$ & 1 & $z 3 * *$ & 0 \\
24 & 6 & 0 & -7 \\
$26_{1}$ & -1 & 8 & 7 \\
$26_{2}$ & -1 & $8 z 3$ & 7 \\
$26_{3}$ & -1 & $8 z 3 * *$ & 7 \\
144 & 0 & 0 & 0 \\
$246_{1}$ & -6 & 12 & 0 \\
$246_{2}$ & -6 & $12 z 3$ & 0 \\
$246_{3}$ & -6 & $12 z 3 * *$ & 0 \\
\hline
\end{tabular}

Because of $7 A-7 B$, the restriction of a character of degree 246 or 247 contains as many copies of 24 as of 26 . As $3 A$ fuses into $3 A$, no $246_{i}$ can be contained in the restriction. If the restriction did not contain any 144 , the value on $3 A$ would be at least $4 \cdot 5+46 \cdot 1=66$, a contradiction. Hence, $144+2 \cdot 24+2 \cdot 26$ is contained in the restriction to ${ }^{3} D_{4}(2)$. Since $3 C$ fuses into $3 A$, we have $2 \cdot 26_{1}$ within the restriction to $K$. But now for this character of degree 244 the value is 16 on $3 C$; therefore we must have $2 \cdot 1_{2}+2 \cdot 1_{3}$ in the restriction, too. This now adds up to 248 .

\subsubsection{Th modulo 3}

The ordinary 248-dimensional representation remains irreducible modulo 3 . In Th, all elements of order 2 and 7 form one conjugacy class. As in the case modulo 2, we consider the maximal subgroup ${ }^{3} D_{4}(2): 3$. We obtain the following table. 


\begin{tabular}{rcc}
\hline $1 A$ & $2 A-2 B$ & $7 A-7 B$ \\
\hline 1 & 0 & 0 \\
25 & -8 & 7 \\
52 & 24 & 0 \\
196 & -24 & 0 \\
\hline
\end{tabular}

Because of $7 A-7 B$, no 25 is contained in the restriction of a faithful character of degree less than 248 . The column $2 A-2 B$ immediately implies that the restriction contains 52 and 196.

\subsubsection{Th modulo 5}

This is analogous to Th modulo 3, only replacing 25 by 26 .

\subsubsection{Th modulo 7}

We know of the existence of a Brauer character of degree 248. We consider the maximal subgroup ${ }^{3} D_{4}(2): 3$. The classes $8 A B$ fuse into $8 A$, and $4 A B$ fuse into $4 A$. We obtain the following table.

\begin{tabular}{rcc}
\hline $1 A$ & $4 A-4 B$ & $8 A-8 B$ \\
\hline 1 & 0 & 0 \\
26 & 8 & 0 \\
52 & 8 & -4 \\
196 & -8 & 4 \\
\hline
\end{tabular}

The column $8 A-8 B$ shows that the restriction of a potential character contains as many copies of 52 as of 196 . The column $4 A-4 B$ now proves the result.

\subsubsection{5. $\mathrm{Fi}_{23}$ modulo 2}

By Fong's lemma (see [15, Theorem VII.8.13]), we know that there is a faithful Brauer character with degree 782 , obtained from the ordinary character of degree 783 . We consider the maximal subgroup $O_{8}^{+}(3): S_{3}$, whose 2-modular character table can be found in [3]. The classes $3 A$ and $3 D$ fuse into $3 B$, and $3 C$ and $3 H$ fuse into $3 A$ of $\mathrm{Fi}_{23}$. We obtain the following table.

\begin{tabular}{rcc}
\hline $1 A$ & $3 A-3 D$ & $3 C-3 H$ \\
\hline 1 & 0 & 0 \\
2 & 0 & 3 \\
298 & 54 & 0 \\
596 & 108 & 84 \\
$780_{1}$ & 0 & -3 \\
$780_{2}$ & 81 & 24 \\
\hline
\end{tabular}

First, $3 A-3 D$ shows that the restriction of a potential character of degree at most 781 does not contain 298,596 or $780_{2}$. But then $3 C-3 H$ proves that the restriction must contain $780_{1}+2$. 


\subsubsection{6. $\mathrm{Fi}_{23}$ modulo 3}

On the one hand, we see the existence of a 253-dimensional representation from [30], which is described in [2]. On the other hand, in the maximal subgroup $2^{11} \cdot \mathrm{M}_{23}$ there is no smaller faithful representation.

\subsubsection{7. $\mathrm{Co}_{1}$ modulo 2}

The reduction modulo 2 of the ordinary character of $2 \cdot \mathrm{Co}_{1}$ of degree 24 yields a faithful 2modular character of $\mathrm{Co}_{1}$. On the other hand, the smallest faithful representation of $3 \cdot \mathrm{Suz}: 2$ in characteristic 2 has degree 24 .

\subsubsection{8. $2 \cdot \mathrm{Co}_{1}$ modulo 3}

There is a restricted ordinary character of degree 24 that restricts to $6 \cdot \mathrm{Suz}: 2$ as $12+12^{\prime}$, which are the only faithful irreducible representations for $2 \cdot \mathrm{Suz}: 2$ with degree less than 24 . The values of 12 on several outer 8 -classes are irrational, and hence 24 is the smallest faithful representation.

\subsubsection{9. $2 \cdot \mathrm{Co}_{1}$ modulo 5 and 7}

There is a restricted ordinary character of degree 24 that remains irreducible when restricted to 6.Suz:2. This 24-dimensional representation is the smallest one for 6·Suz:2.

\subsubsection{Co 1 modulo 3,5 and 7}

On the one hand, the degree of the smallest faithful representation for the maximal subgroup $2^{11}: \mathrm{M}_{24}$ is 276 . On the other hand, there is a restricted ordinary representation of degree 276.

\subsubsection{1. $\mathrm{J}_{4}$ modulo 2}

John Thompson has shown that the minimal faithful degree of $\mathrm{J}_{4}$ in characteristic 2 is at least 112, and that it is at least 1333 in any other characteristic (see [1, p. 61]). Since we have not found a published proof of Thompson's result, we give our own proof below.

Benson, Conway, Norton, Parker and Thackray constructed $\mathrm{J}_{4}$ as a group of $(112 \times 112)$ matrices over the field with two elements (see [1]). Thus we have only to prove that no smaller faithful representation exists in characteristic 2 .

The group $\mathrm{J}_{4}$ has a maximal subgroup isomorphic to $U_{3}(11): 2$, whose ordinary and 2modular table can be found in [20]. The restriction to $U_{3}(11): 2$ proves that the minimal degree is $110+a$, where $a \in\{0,1,2\}$ with value $2+a$ on $3 A$ which fuses into $3 A$, the only 3-class of $\mathrm{J}_{4}$.

Next, we consider $H:=11_{+}^{1+2}:\left(5 \times 2 S_{4}\right)$. Its ordinary character table can be taken from [3]. As $H$ is soluble, the 2-modular character table of $H$ can be computed algorithmically from the ordinary character table using the theorem of Fong and Swan (see, for example, [24, Chapter 5, Theorem 7.5]). That is, the set of irreducible Brauer characters in this case consists of those restricted ordinary characters that cannot be written as the sum of two restricted characters. When restricting a potential 110 or 111 to $H$, we see that the only faithful characters of $H$ being contained in the restriction are characters of degree 110 . However, their values on $3 A$ are 5 and -10 . This immediately implies that there cannot be a 110 or a 111. 


\subsubsection{2. $\mathrm{J}_{4}$ modulo 3}

There is a 1333-dimensional faithful representation, because there is an ordinary one. We consider $2^{11}: \mathrm{M}_{24}$, which is a maximal subgroup of $\mathrm{J}_{4}$. The ordinary and the 3-modular character table can be found in [3]. The 3-modular table can be obtained from the Brauer character tables of the inertia factor groups $2^{4}: A_{8}$ and $\mathrm{M}_{12}: 2$ with the help of Clifford theory; see, for example, [24, Chapter 3, Section 3]. There are only three irreducible faithful 3-modular characters for $2^{11}: \mathrm{M}_{24}$ of degree less than 1333, namely 759 and 1288 (the latter occurring twice). Therefore, we have to consider characters of the factor group $\mathrm{M}_{24}$ of degrees up to 574 . The classes $2 B C D$ fuse into $2 A$ of $\mathrm{J}_{4}$, and the factor fusion maps $2 B$ to $1 A, 2 C$ to $2 A$, and $2 D$ to $2 B$. We obtain the following table.

\begin{tabular}{rrr}
\hline $1 A$ & $2 B-2 C$ & $2 B-2 D$ \\
\hline 1 & 0 & 0 \\
22 & 16 & 24 \\
45 & 48 & 40 \\
231 & 224 & 240 \\
252 & 224 & 240 \\
483 & 448 & 480 \\
759 & -80 & -24 \\
$1288_{1}$ & -48 & -40 \\
$1288_{2}$ & -48 & 24 \\
\hline
\end{tabular}

If the restriction of a representation of $\mathrm{J}_{4}$ of degree at most 1333 contained a 759 , then the column for $2 B-2 D$ would imply that the representation must contain $759+22$, contradicting column $2 B-2 C$. Therefore, $1288_{1}$ must be contained. Again, $2 B-2 D$ implies that the restriction contains $1288_{1}+45$ of degree 1333 .

\subsubsection{3. $\mathrm{J}_{4}$ modulo 11}

Here we use the 11-modular character table of the same maximal subgroup $2^{11}: \mathrm{M}_{24}$ of $\mathrm{J}_{4}$. The relevant character values and differences between the character values are given in the following table.

\begin{tabular}{rrr}
\hline $1 A$ & $2 B-2 C$ & $2 B-2 D$ \\
\hline 1 & 0 & 0 \\
23 & 16 & 24 \\
45 & 48 & 40 \\
229 & 208 & 216 \\
231 & 224 & 240 \\
253 & 240 & 264 \\
482 & 448 & 480 \\
759 & -80 & -24 \\
12881 & -48 & -40 \\
$1288_{2}$ & -48 & 24 \\
\hline
\end{tabular}

The same argument as in the 3-modular case, replacing 22 by 23 , proves the result. 


\subsubsection{4. $\mathrm{F}_{3}+$ modulo 2}

See above.

\subsubsection{5. $\mathrm{F}_{3}+$ modulo 3}

In [25], S. Norton proves the existence of a 781-dimensional faithful representation. We restrict a representation to the maximal subgroup $O_{10}^{-}(2)$, whose 3-modular character table is completely known and is available in [3]. The classes $2 B$ and $2 C$ both fuse into $2 A$. It follows that the restriction contains 595 and 186, which add up to 781 .

\subsubsection{6. $\mathrm{F}_{3+}$ modulo 5 and 7}

Again, we consider the maximal subgroup $3^{7} \cdot O_{7}(3)$ and its classes $3 D F G N O$, which all fuse into $3 D$ of $\mathrm{F}_{3+}$. The factor fusion map sends $3 D F G$ to $3 A$, and $3 N O$ to $3 C$. Therefore, the columns for $3 F-3 G, 3 N-3 O$ and $3 D-3 G$ are zero for all characters derived from $\mathrm{O}_{7}$ (3). Later on, we use the fact that $2 \mathrm{~A}$ and $2 \mathrm{C}$ both fuse into $2 \mathrm{~B}$. The ordinary and modular character tables can be found in [3]. We obtain the following table.

\begin{tabular}{rrrrc}
\hline $1 A$ & $2 A-2 C$ & $3 F-3 G$ & $3 N-3 O$ & $3 D-3 G$ \\
\hline 728 & -8 & -81 & 27 & -54 \\
756 & -12 & 81 & -27 & -27 \\
$3640_{1}$ & 24 & -162 & 54 & -27 \\
$3640_{2}$ & 24 & 81 & 54 & -108 \\
4212 & 0 & -243 & 0 & -81 \\
4368 & 16 & 243 & 0 & 81 \\
$7280_{1}$ & -16 & 162 & 27 & 108 \\
$7280_{2}$ & -16 & 405 & 27 & 27 \\
\hline
\end{tabular}

We restrict a non-trivial character of degree at most 8671 . If the restriction contained a $7280_{2}$, then it would include a 4212 or $3640_{1}$, or five copies of 728 , because of $3 F-3 G$, which adds up to more than 8671 . Likewise, if there were a $7280_{1}$, there would be two copies of 728, which gives a contradiction. Therefore, $3 D-3 G$ proves that the restriction contains a 4368 . Next, $3 F-3 G$ shows that we have 4212 , too, as $3640_{1}+728+4368>8671$ and the possibility of $4368+3 \cdot 728$ yields another three copies of 756 because of $3 \mathrm{~N}-3 \mathrm{O}$. Hence, we have $8580:=4368+4212$. We still have to consider the characters of degree 1,78 and 91, derived from the factor group $\mathrm{O}_{7}(3)$. We obtain the following values.

\begin{tabular}{rc}
\hline $1 A$ & $2 A-2 C$ \\
\hline 1 & 0 \\
78 & -32 \\
91 & -16 \\
8580 & 16 \\
\hline
\end{tabular}

This table immediately implies that the restriction contains 91, which now takes it up to 8671. 


\subsubsection{7. $3 \cdot \mathrm{F}_{3}+$ modulo $2,5,7$}

We know of the existence of a Brauer character of degree 783. In the maximal subgroup $3 \times \mathrm{Fi}_{23}$, the irreducible Brauer characters of degrees less than 783 have degrees 1 and 782 . As the degree has to be divisible by 3 , we get 783 .

\subsubsection{B modulo 2}

In [29], R. A. Wilson constructed a 4370-dimensional representation for B over the field with two elements. We restrict a non-trivial representation of degree at most 4370 to the subgroup $O_{8}^{+}(3): S_{4}$. The classes $3 A, 3 E$ and $3 G$ of $O_{8}^{+}(3): S_{4}$ all fuse into $3 B$. We obtain the following table.

\begin{tabular}{rcr}
\hline $1 A$ & $3 A-3 E$ & $3 A-3 G$ \\
\hline 1 & 0 & 0 \\
2 & 0 & 3 \\
298 & 45 & 54 \\
596 & 90 & 111 \\
1560 & 108 & 102 \\
2808 & -108 & -105 \\
\hline
\end{tabular}

Because of $3 A-3 E$, the restriction of a faithful Brauer character for B of degree 4370 must contain a 2808 . If the restriction did not contain any 1560 , then it would contain only 2808, 298, 596, 1 and 2. But then 298 occurs an even number of times. Hence, 2808 has to occur five times to obtain zero on $3 A-3 E$. Therefore, the restriction to $O_{8}^{+}(3): S_{4}$ contains $2808+1560$. Next, $3 A-3 G$ proves that the restriction contains a 2 . This now adds up to 4370 .

\subsubsection{9. $2 \cdot \mathrm{B}$ modulo 3}

The Thompson group Th has six irreducible 3-modular characters whose degrees do not exceed 96256 . This follows from (unpublished) work of Jürgen Müller and others (Hiss, Lux, Lübeck and Neunhöffer). The following table gives the values of these six characters on certain conjugacy classes, whose ATLAS names are given in the first row of the table.

\begin{tabular}{rrrrrr}
\hline $1 A$ & $2 A$ & $4 B$ & $8 A$ & $14 A$ & $20 A$ \\
\hline 1 & 1 & 1 & 1 & 1 & 1 \\
248 & -8 & 0 & 0 & -1 & 0 \\
3875 & 35 & -5 & 3 & 0 & 0 \\
27000 & 120 & 0 & 0 & 1 & 0 \\
30132 & -76 & 4 & -4 & 1 & -1 \\
85746 & -14 & 10 & 2 & 0 & 0 \\
\hline
\end{tabular}

The five classes $2 A, 4 B, 8 A, 14 A$ and $20 A$ fuse into conjugacy classes of $\mathrm{B}$, whose inverse images in $2 \cdot B$ are single conjugacy classes of $2 \cdot B$. Hence all faithful characters have value 0 on these classes. Let $\varphi$ be the restriction to Th of a faithful 3-modular character of $2 \cdot \mathrm{B}$ of degree at most 96256 . Then $\varphi$ has value 0 on these classes as well. 


\section{Degrees of minimal faithful Brauer characters}

Adding the $8 A$-column twice to the $4 B$-column, we obtain the next table.

\begin{tabular}{rc}
\hline $1 A$ & $4 B+2 \times 8 A$ \\
\hline 1 & 3 \\
248 & 0 \\
3875 & 1 \\
27000 & 0 \\
30132 & -4 \\
85746 & 14 \\
\hline
\end{tabular}

This shows that 85746 cannot be a constituent of $\varphi$. Looking at class $20 \mathrm{~A}$, we see that 1 and 30132 occur the same number of times in $\varphi$.

We obtain the following table.

\begin{tabular}{rrrr}
\hline $1 A$ & $2 A$ & $8 A$ & $14 A$ \\
\hline 248 & -8 & 0 & -1 \\
3875 & 35 & 3 & 0 \\
27000 & 120 & 0 & 1 \\
30133 & -75 & -3 & 2 \\
\hline
\end{tabular}

From $8 A$, we conclude that 3875 and 30133 occur the same number of times in $\varphi$.

We obtain the following table.

\begin{tabular}{rrr}
\hline $1 A$ & $2 A$ & $14 A$ \\
\hline 248 & -8 & -1 \\
27000 & 120 & 1 \\
34008 & -40 & 2
\end{tabular}

It is now easy to conclude that 248 must occur at least five times in $\varphi, 27000$ at least once, and 34008 at least twice. Thus the degree of $\varphi$ is at least $5 \cdot 248+27000+2 \cdot 34008=96256$.

\subsubsection{B modulo 3}

Let $\varphi$ be a faithful character of degree less than 4371. We restrict to the subgroup $F_{4}(2)$. We have the following values.

\begin{tabular}{rrrrc}
\hline \multicolumn{1}{c}{$1 A$} & $2 A-2 B$ & $2 B-2 C$ & $2 D$ & $4 E-4 F$ \\
\hline 1 & 0 & 0 & 1 & 0 \\
833 & 0 & -128 & 1 & 32 \\
$1105_{1}$ & 256 & -128 & 17 & -32 \\
$1105_{2}$ & -256 & 128 & 17 & -32 \\
1326 & 0 & 128 & -18 & 32 \\
1377 & 0 & 128 & 33 & 32 \\
\hline
\end{tabular}


This table is derived from the part of the 3-modular character table of $F_{4}(2)$ containing all the irreducible characters of degree less than 4371. For a proof for this, see [8]. Note that all 3-modular characters of $F_{4}(2)$ of degrees smaller than 4371 are liftable, so that the corresponding character values can be found in GAP.

The classes $2 A, 2 B$ and $2 C$ all fuse into the conjugacy class $2 B$ of $\mathrm{B}$; in addition, $4 E$ and $4 F$ fuse into $4 G$. Furthermore, $2 D$ maps to $2 D$. By looking at the column $4 E-4 F$, we see that $\varphi$ must contain one of the $1105 \mathrm{~s}$. By looking at $2 A-2 B$, we also see that it follows that then the other 1105 is also a constituent of $\varphi$. Again, by looking at $4 E-4 F$, we find that two of the other three non-trivial irreducible characters must be contained in $\varphi$. The degree of $\varphi$ and the three 2-classes show that 833 and 1326 must be contained in $\varphi$. It follows that $\varphi(1) \geqslant 4369$, and $\varphi(2 D) \leqslant 18$.

Now look at the 3-modular degrees of Th. Only 248 and 3875 are non-trivial irreducible characters that can be constituents of $\varphi$. Moreover, class $2 A$ of Th fuses into class $2 D$ of B. It follows that $\varphi$ contains 3875 and 248, the latter at least twice. But $3875+2 \cdot 248=4371$, a contradiction.

\subsubsection{B modulo 5, 7, 19 and 47}

Similarly to the case B modulo 2, we will prove that the restriction of a potential character of B of degree less than 4371 to $O_{8}^{+}(3): S_{4}$ contains one irreducible Brauer character of degree 2808, and one of degree 1560. We obtain this result in characteristic 5 from the next table.

\begin{tabular}{|c|c|c|c|c|}
\hline $1 A$ & $2 B-2 F$ & $3 A-3 E$ & $3 A-3 G$ & $4 D$ \\
\hline $1_{1}$ & 2 & 0 & 0 & 1 \\
\hline $1_{2}$ & 0 & 0 & 0 & 1 \\
\hline 2 & 2 & 0 & 3 & 2 \\
\hline 31 & 4 & 0 & 3 & -1 \\
\hline $3_{2}$ & 2 & 0 & 3 & -1 \\
\hline $300_{1}$ & 2 & 45 & 57 & 20 \\
\hline $300_{2}$ & 22 & 45 & 57 & 20 \\
\hline 600 & 24 & 90 & 114 & 40 \\
\hline $900_{1}$ & 26 & 135 & 171 & -20 \\
\hline $900_{2}$ & 46 & 135 & 171 & -20 \\
\hline $1560_{1}$ & 2 & 108 & 102 & 68 \\
\hline $1560_{2}$ & 46 & 108 & 102 & 68 \\
\hline $1560_{3}$ & 30 & 108 & 102 & -68 \\
\hline $1560_{4}$ & 18 & 108 & 102 & -68 \\
\hline $2454_{1}$ & 22 & 243 & 267 & 46 \\
\hline $2454_{2}$ & 86 & 243 & 267 & 46 \\
\hline $2454_{3}$ & 22 & 243 & 267 & 134 \\
\hline $2454_{4}$ & 86 & 243 & 267 & 134 \\
\hline $2808_{1}$ & 4 & -108 & -105 & 120 \\
\hline $2808_{2}$ & -20 & -108 & -105 & 120 \\
\hline
\end{tabular}




\section{Degrees of minimal faithful Brauer characters}

The result in characteristic 7 comes from the table below:

\begin{tabular}{rrrrr}
\hline $1 A$ & $2 B-2 F$ & $3 A-3 E$ & $3 A-3 G$ & $4 D$ \\
\hline $1_{1}$ & 0 & 0 & 0 & 1 \\
$1_{2}$ & 2 & 0 & 0 & 1 \\
2 & 2 & 0 & 3 & 2 \\
$3_{1}$ & 2 & 0 & 3 & -1 \\
$3_{2}$ & 4 & 0 & 3 & -1 \\
$299_{1}$ & 2 & 45 & 57 & 19 \\
$299_{2}$ & 20 & 45 & 57 & 19 \\
598 & 22 & 90 & 111 & 38 \\
$897_{1}$ & 24 & 135 & 168 & -19 \\
$897_{2}$ & 42 & 135 & 168 & -19 \\
$1560_{1}$ & 2 & 108 & 102 & 68 \\
$1560_{2}$ & 46 & 108 & 102 & 68 \\
$1560_{3}$ & 30 & 108 & 102 & -68 \\
$1560_{4}$ & 18 & 108 & 102 & -68 \\
$2457_{1}$ & 24 & 243 & 270 & 49 \\
$2457_{2}$ & 90 & 243 & 270 & 49 \\
$2457_{3}$ & 24 & 243 & 270 & 133 \\
$2457_{4}$ & 90 & 243 & 270 & 133 \\
$2808_{1}$ & 4 & -108 & -105 & 120 \\
$2808_{2}$ & -20 & -108 & -105 & 120 \\
\hline
\end{tabular}

and in characteristic 19 and 47 from the following table.

\begin{tabular}{rrrrr}
\hline $1 A$ & $2 B-2 F$ & $3 A-3 E$ & $3 A-3 G$ & $4 D$ \\
\hline $1_{1}$ & 0 & 0 & 0 & 1 \\
$1_{2}$ & 2 & 0 & 0 & 1 \\
2 & 2 & 0 & 3 & 2 \\
$3_{1}$ & 2 & 0 & 3 & -1 \\
$3_{2}$ & 4 & 0 & 3 & -1 \\
$300_{1}$ & 2 & 45 & 57 & 20 \\
$300_{2}$ & 22 & 45 & 57 & 20 \\
600 & 24 & 90 & 114 & 40 \\
$900_{1}$ & 26 & 135 & 171 & -20 \\
$900_{2}$ & 46 & 135 & 171 & -20 \\
$1560_{1}$ & 2 & 108 & 102 & 68 \\
$1560_{2}$ & 46 & 108 & 102 & 68 \\
$1560_{3}$ & 30 & 108 & 102 & -68 \\
$1560_{4}$ & 18 & 108 & 102 & -68 \\
$2457_{1}$ & 24 & 243 & 270 & 49 \\
$2457_{2}$ & 90 & 243 & 270 & 49 \\
$2457_{3}$ & 24 & 243 & 270 & 133 \\
$2457_{4}$ & 90 & 243 & 270 & 133 \\
$2808_{1}$ & 4 & -108 & -105 & 120 \\
$2808_{2}$ & -20 & -108 & -105 & 120 \\
\hline
\end{tabular}


The classes $2 B$ and $2 F$ both fuse into $2 D$ of $B$ and $4 D$ fuses into $4 B$. First, $2 B-2 F$ shows that the restriction must contain $2808_{2}$. Next, $3 A-3 E$ proves that $1560_{i}$ is contained. In turn, the column for $2 B-2 F$ proves that the restriction must be $2808_{2}+1560_{4}$. Finally, $3 A-3 G$ shows that a copy of 2 is contained. This character leads to the value 54 on $4 B$ in $\mathrm{B}$.

Now we consider the subgroup $F_{4}(2)$. We have the following table.

\begin{tabular}{rrrrc}
\hline \multicolumn{1}{c}{$1 A$} & $2 A-2 B$ & $2 B-2 C$ & $4 A$ & $4 E-4 F$ \\
\hline 1 & 0 & 0 & 1 & 0 \\
833 & 0 & -128 & 25 & 32 \\
$1104_{1}$ & 256 & -128 & 8 & -32 \\
$1104_{2}$ & -256 & 128 & 24 & -32 \\
1326 & 0 & 128 & -10 & 32 \\
1377 & 0 & 128 & 9 & 32 \\
\hline
\end{tabular}

This table is obtained from the part of the 7-modular character table of $F_{4}(2)$ containing all the irreducible Brauer characters of degree less than 4371; the proof for this can be found in [8]. As in the 3-modular case the restriction of a non-trivial character of degree 4370 must contain $833,1104_{1}, 1104_{2}$ and 1326 . Hence the degree is at least 4367 , and the value on $4 A$ is at most 50 . But $4 A$ fuses into $4 B$ of $\mathrm{B}$, which contradicts the result obtained from $O_{8}^{+}(3): S_{4}$.

The result for the characteristics 5, 19 and 47 can be achieved in the same way: the characters of degree 1104 have to be replaced by restricted ordinary characters of degree 1105 .

\subsubsection{2. $2 \cdot B$ modulo 5}

This proof is taken from unpublished work of R. Parker. The Fischer group $\mathrm{Fi}_{23}$ has eight irreducible 5-modular characters whose degrees do not exceed 96256 . The following table gives their values on certain conjugacy classes, whose ATLAS names are given in the first row of the table. The 5-modular table is given in [10], and is also available through GAP [3].

\begin{tabular}{rrrrrrr}
\hline $1 A$ & $2 A$ & $2 C$ & $3 A$ & $3 C$ & $4 A$ & $4 C$ \\
\hline 1 & 1 & 1 & 1 & 1 & 1 & 1 \\
782 & 78 & 14 & -1 & 26 & 6 & 6 \\
3588 & -572 & 4 & 78 & 51 & -28 & 4 \\
5083 & 923 & -37 & 169 & 34 & 27 & -5 \\
25806 & -2354 & -50 & 183 & 75 & -42 & -10 \\
30106 & 3354 & 154 & 352 & 82 & 50 & 18 \\
60996 & 1924 & 68 & -78 & 165 & 36 & 4 \\
76637 & 7293 & 93 & 740 & -70 & 41 & -7 \\
\hline
\end{tabular}

The four classes $2 A, 2 C, 4 A$ and $4 C$ fuse into the conjugacy classes $2 A, 2 D, 4 D$ and $4 G$ of $\mathrm{B}$, whose inverse images in $2 \cdot \mathrm{B}$ are single conjugacy classes in $2 \cdot \mathrm{B}$. The classes $3 A$ and $3 C$ fuse into conjugacy class $3 A$ of $B$. It follows that all the faithful characters have value 0 on those classes of $2 \cdot B$ to which the classes $2 A, 2 C, 4 A$ and $4 C$ are mapped. Also, $3 A$ and $3 C$ fuse in $2 \cdot \mathrm{B}$.

Let $\varphi$ be the restriction to $\mathrm{Fi}_{23}$ of a faithful 5-modular character of $2 \cdot \mathrm{B}$, of degree at most 96256. Then $\varphi$ has value 0 on the classes $2 A, 2 C, 4 A$ and $4 C$, and equal values on $3 A$ and $3 C$. 
We find the following values.

\begin{tabular}{rc}
\hline $1 A$ & $3 A-3 C+10 \times 4 C$ \\
\hline 1 & 10 \\
782 & 33 \\
3588 & 67 \\
5083 & 85 \\
25806 & 8 \\
30106 & 450 \\
60996 & -203 \\
76637 & 740 \\
\hline
\end{tabular}

This means that 60996 is contained in $\varphi$. We have $\varphi(1)-60996 \leqslant 35260$. Thus 76637 cannot be a constituent of $\varphi$.

If 30106 were in $\varphi$, then the value on $2 A$ would be positive. Hence 30106 is not a constituent of $\varphi$.

If 25806 is not in $\varphi$, then 5083 must be in $\varphi$ (look at $4 C$ ). This leaves 30177 . Now the value on $4 A$ is 63 , so we must have $3 \cdot 3588$. This leaves 19413 . Now $\varphi$ is stuck positive on $4 C$, a contradiction.

It follows that 25806 is contained in $\varphi$, which leaves dimension 9454 . We must have 5083 as well, to get the value 0 on $2 C$. This leaves dimension 4371 . The current value on $2 A$ is positive, namely 493 , and so we need 3588 as well. This leaves dimension 783 , and the value -79 on $2 A$. Looking at $2 A$ and $2 C$, we see that 782 and 1 must occur in $\varphi$. So $\varphi$ has degree at least $1+782+3588+5083+25806+60996=96256$. This proves the result for the prime 5 .

\subsubsection{3. $2 \cdot$ B modulo $7,13,31$ and 47}

We proceed as above. For $p \in\{7,13,31,47\}$, there are seven irreducible $p$-modular characters of $\mathrm{Fi}_{23}$ with degree not exceeding 96256. In the cases $p=7$ and $p=13$, the Brauer trees are given in [9]. In the two other cases, $p$ does not divide the order of $\mathrm{Fi}_{23}$. The character values on the classes $1 A, 2 A, 2 C, 3 A, 3 C, 4 A$ and $4 C$ are given in the table below. (These values are the same for all $p$.) The proof now proceeds exactly as in the case $p=5$.

\begin{tabular}{rrrrrrr}
\hline $1 A$ & $2 A$ & $2 C$ & $3 A$ & $3 C$ & $4 A$ & $4 C$ \\
\hline 1 & 1 & 1 & 1 & 1 & 1 & 1 \\
782 & 78 & 14 & -1 & 26 & 6 & 6 \\
3588 & -572 & 4 & 78 & 51 & -28 & 4 \\
5083 & 923 & -37 & 169 & 34 & 27 & -5 \\
25806 & -2354 & -50 & 183 & 75 & -42 & -10 \\
30888 & 3432 & 168 & 351 & 108 & 56 & 24 \\
60996 & 1924 & 68 & -78 & 165 & 36 & 4 \\
\hline
\end{tabular}

Acknowledgements. I thank Richard Parker, Jürgen Müller and Gerhard Hiss, who allowed me to include their unpublished work on, respectively: determining the minimal degrees for $2 \cdot \mathrm{B}$ modulo 3, 5, 7 and 13; constructing the irreducible 3774-dimensional representation of $\mathrm{F}_{3+}$; and proving its minimality. 


\section{References}

1. D. J. Benson, 'The simple group $\mathrm{J}_{4}$ ', $\mathrm{PhD}$ thesis, University of Cambridge, 1981. 134

2. J .H. Conway, R. T. Curtis, S. P. Norton, R. A. Parker and R. A. Wilson, AtLAS of finite groups (Clarendon Press, 1985). 122, 126, 127, 134

3. The GAP Group, 'GAP - groups, algorithms, and programming', Version 4.4, 2004, http://www.gap-system.org. 125, 126, 128, 133, 134, 135, 136, 141

4. R. L. Griess, JR. and S. D. SMith, 'Minimal dimensions for modular representations of the Monster', Comm. Algebra 22 (1994) 6279-6294. 125

5. A. Henke, G. Hiss and J. Müller, 'The 7-modular decomposition matrices of the sporadic O’Nan group', J. London Math. Soc. (2) 60 (1999) 58-70. 125

6. S. Hensing, '5-modulare Zerlegungszahlen der sporadischen einfachen Gruppe $\mathrm{Co}_{1}$ und ihrer Überlagerungsgruppe 2.Co1', Diplomarbeit, Universität Heidelberg, 1993. 125

7. G. Hiss, 'The 3-modular characters of the Rudvalis group and its covering group', Math. Comput. 62 (1994) 851-863. 125

8. G. Hiss, 'Decomposition matrices of the Chevalley group $F_{4}(2)$ and its covering group', Comm. Algebra 25 (1997) 2539-2555. 139, 141

9. G. Hiss and K. Lux, Brauer trees of sporadic groups (Clarendon Press, Oxford, 1989). 125,142

10. G. Hiss and K. Lux, with an appendix by T. Breuer, 'The 5-modular characters of the sporadic simple Fischer groups $\mathrm{Fi}_{22}$ and $\mathrm{Fi}_{23}$ ', Comm. Algebra 22 (1994) 3563-3590. 125,141

11. G. Hiss and J. Müller, 'The 5-modular characters of the sporadic simple Rudvalis group and its covering group', Comm. Algebra 23 (1995) 4633-4667. 125

12. G. Hiss and D. L. White, 'The 5-modular characters of the covering group of the sporadic simple Fischer group $\mathrm{Fi}_{22}$ and its automorphism group', Comm. Algebra 22 (1994) 3591-3611. 125

13. G. Hiss, C. JANSEN, K. LuX and R. PARKER, 'Computational modular character theory', 1992, http://www.math.rwth-aachen.de/LDFM/homes/MOC/CoMoChaT/. 125

14. B. Huppert, Character theory of finite groups (Walter de Gruyter \& Co., Berlin, 1998). 126

15. B. Huppert and N. Blackburn, Finite groups II (Springer, Berlin/Heidelberg/New York, 1982). 130, 133

16. C. JANSEN, Ein Atlas 3-modularer Charaktertafeln, Dissertation, RWTH Aachen, 1995 (Marchal-und Matzenbacher-Wiss.-Verl., Krefeld, 1995). 125

17. C. JANSEN and J. MüLleR, 'The 3-modular decomposition numbers of the sporadic simple Suzuki group', Comm. Algebra 25 (1997) 2437-2458. 125

18. C. JANSEN and R. A. WILSON, 'The minimal faithful 3-modular representation for the Lyons group', Comm. Algebra 24 (1996) 873-879. 131

19. C. JANSEN and R. A. WILSON, 'The 2-modular and 3-modular decomposition numbers for the sporadic simple O'Nan group and its triple cover', J. London Math. Soc. (2) 57 (1998) 71-90. 125 
20. C. JANSEN, K. Lux, R. PARKER and R. Wilson, An atlas of Brauer characters (Clarendon Press, Oxford, 1995). 122, 125, 126, 134

21. W. Meyer, W. Neutsch and R. Parker, 'The minimal 5-representation of Lyons' sporadic group', Math. Ann. 272 (1985) 29-39. 131

22. J. MülLER, 'The 5-modular decomposition matrix of the sporadic simple Conway group $\mathrm{Co}_{3}$ ', Proc. 1998 International Symposium on Symbolic and Algebraic Computation (Rostock) (ACM, New York, 1998) 179-185 (electronic). 125

23. J. Müller and J. Rosenboom, 'Condensation of induced representations and an application: The 2-modular decomposition numbers of $\mathrm{Co}_{2}$ ', Computational methods for representations of groups and algebras (Essen, 1997), Progr. Math. 173 (Birkhäuser, Basel, 1999) 309-321. 125

24. H. NagaO and Y. Tsushima, Representations of finite groups (Academic Press, 1989). $122,126,134,135$

25. S. Norton, 'On the group Fi 24 ', Geom. Dedicata 25 (1988) 483-501. 136

26. A. J. E. RyвA, 'Calculation of the 7-modular characters of the Held group', J. Algebra 117 (1988) 240-255. 125

27. I. A. I. Suleiman and R. A. Wilson, 'The 2-modular characters of Conway's group $\mathrm{Co}_{2}$ ', Math. Proc. Camb. Philos. Soc. 116 (1994) 275-283. 125

28. I. A. I. Suleiman and R. A. Wilson, 'The 2-modular characters of Conway's third group $\mathrm{Co}_{3}$ ', J. Symbolic Comput. 24 (1997) 493-506. 125

29. R. A. Wilson, 'A new construction of the Baby Monster and its applications', Bull. London Math. Soc. 25 (1993) 431-437. 137

30. R. A. WiLson, et al., 'AtLas of finite group representations', 2004, http://brauer.maths.qmul.ac.uk/Atlas/. 126, 129, 134

Christoph Jansen christoph.jansen@itergo.com

ITERGO

Informationstechnologie $\mathrm{GmbH}$

Victoriaplatz 1

40477 Düsseldorf

Germany 